\title{
Mutual Coupling Reduction of MIMO Antennas using Parasitic Elements for Wireless Communications
}

\author{
O.F. Ahmed \\ MSc student at Ain Shams \\ University, Cairo, Egypt
}

\author{
R.S. Ghoname \\ Electronic research institute, \\ Cairo, Egypt
}

\author{
A. A. Zekry \\ Faculty of engineering Ain \\ shams university, Cairo, Egypt
}

\begin{abstract}
A compact two element MIMO (Multiple Input Multiple Output) system is proposed using $\mathrm{H}$-shape antenna with parasitic elements employing polarization diversity. The proposed MIMO system offers improved bandwidth; return loss, separation between antenna elements and isolation characteristics. The system resonates at $2.36 \mathrm{GHz}$ and $5.2 \mathrm{GHz}$ frequencies with VSWR $\leq 2$, which can be used for $4 \mathrm{G} \&$ WiMAX applications. The simulation results of return loss, mutual coupling, correlation coefficient and gain are presented. The design is performed by using Ready-made software package Zeland- IE3D. The antennas are fabricated using thin film and photolithographic technique and measured using the Vector Network Analyzer. Good agreements were found between the simulated and measured results.
\end{abstract}

\section{General Terms}

MIMO antennas, Frequency characteristics, mutual coupling, correlation coefficient, parasitic elements.

\section{Keywords}

MIMO systems, SISO systems, Polarization diversity, WiMAX, mutual coupling.

\section{INTRODUCTION}

In the future, wireless communications will require higher data rate for various mobile services. MIMO (Multiple Input Multiple Output) technology plays a key role for attaining improved data rates and accuracy. The technique of improving the channel capacity with increasing the number of antennas at the transmitter and receiver was first predicted by Foschini in [1]. This technique gives an ample opportunity for the researchers and academicians to explore the ways of enhancing the data rates. However, when multiple antennas are brought at closer spacing the technical challenges are more pronounced compared to a SISO (Single Input Single Output) system. Hence, the basic aim of MIMO antennas design is to minimize the correlation between the multiple signals [2]. The correlation between the received signals is caused by mutual coupling of the transmitting MIMO antennas. By calculating the mutual coupling, one can analyze the electromagnetic field interactions that exist between antenna elements of a MIMO system. Higher mutual coupling may result in higher correlation coefficients which affects the transmission capacity of MIMO systems [3]. The mutual coupling mainly depends on the distance between the antenna elements. By increasing the distance between the elements of the antennas, the mutual coupling can be reduced. However, the distance between the antennas cannot be made too large, since MIMO systems have their major applications in Mobile terminals, laptops, MODEMs, WLAN Access Points etc., where miniaturization is the main concern. The distance between antenna elements in practice cannot be extended beyond certain level which limits the use of spatial diversity to achieve the desired spectral efficiencies and transmission qualities. In addition to increasing the distance between adjacent antenna elements, various methods have been proposed to enhance isolation among the elements [4-6]. Among those three methods is the polarization diversity.

In this paper, the orthogonal polarization concept is applied to achieve isolation between adjacent antenna elements. We enhanced the isolation by adding parasitic elements for each antenna element, in this way we could realize relatively high isolation with minimum separation between antennas elements.

This paper is organized such that section 2 introduces the antenna design and deals with the proposed two elements MIMO antenna with enhanced isolation using parasitic elements. Section 3 concludes the paper.

\section{ANTENNA DESIGN}

A normal $\mathrm{H}$-shape patch antenna is proposed, which can be used for $4 \mathrm{G} \&$ WiMAX application. We use the $\mathrm{H}$ - shape due to its compactness and small size, the area of the proposed shape equal $(21 * 18) \mathrm{mm}^{2}$. The proposed antenna is designed by using FR-4 substrate with the following parameters; dielectric constant $(\varepsilon \mathrm{r}=4.65)$, substrate height $(\mathrm{h}=1.6 \mathrm{~mm})$, loss $\operatorname{tangent}(\tan \delta=0.02)$, and its dimensions as shown in Fig.1.

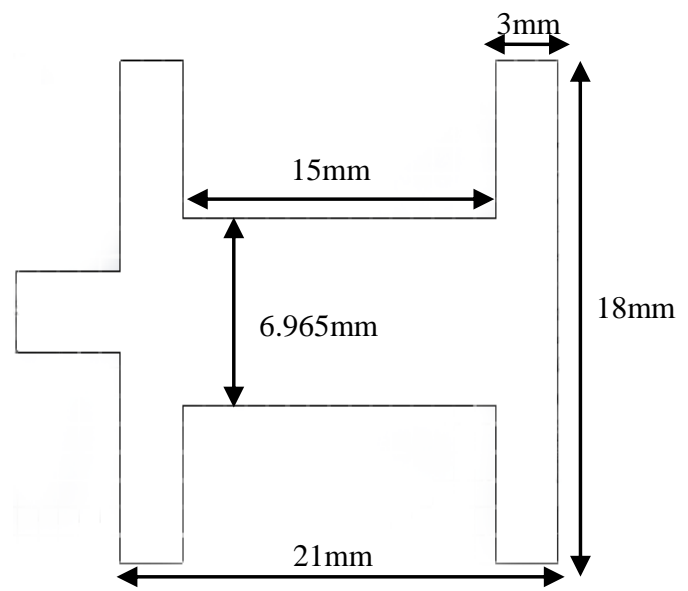

Fig1. H- Shape antenna geometry

\subsection{TWO ELEMENTS MIMO ANTENNAS WITH PARASITIC ELEMENTS BY EMPLOYING POLARIZATION DIVERSITY}

The main design criteria for MIMO system engineers in developing small electronic modules is mutual coupling, which mainly arises due to the smaller spacing between the elements. However, when multiple antennas are involved at closer spacing, the design issues are more complicated compared to a SISO (Single Input Single Output) system. The mutual coupling mainly depends on the distance between the elements of a MIMO system. By increasing the distance 
between the antenna elements, the mutual coupling can be reduced. However, the distance between the elements cannot be maintained too large, since MIMO systems have their major mobile applications, where miniaturization is the main requirements. Hence, another means of achieving low mutual coupling is employing the diversity concept [7].

In this paper, two H-shaped microstrip antennas with parasitic elements employing and orthogonally polarized are shown in Fig 2. To achieve orthogonal polarization, one element is rotated to $90^{\circ}$ with respect to its adjacent element. The separation between the elements is $3 \mathrm{~mm}$ which is $0.096 \lambda \mathrm{g}$. This separation is much smaller compared to the conventional arrays antenna.

To reduce the mutual coupling between the diversity polarized $\mathrm{H}$ - shaped antennas, we add parasitic elements for each antenna. These parasitic elements make a double coupling path and they can create a reverse coupling which reduces the mutual coupling [8].

The scattering parameters of the antenna system are calculated using Zeland Simulator. Fig.3 (a) shows the reflection coefficients S11 and S22 of the antenna elements as a function of frequency with and without the parasitic elements. Fig3 (b) displays the mutual coupling as a function of frequency for the proposed structure with and without parasitic elements and with diversity polarization. It is clear from these figures that the reflection coefficient of the antenna with parasitic elements has an additional resonance frequency at $2.36 \mathrm{GHz}$ besides the original resonance at $5.2 \mathrm{GHz}$. The mutual coupling at the resonance frequency of the original antenna is $-26 \mathrm{~dB}$. With the addition of parasitic elements, we improve the mutual coupling between the MIMO antennas elements to $-37 \mathrm{~dB}$ which corresponds to an improvement up to $70.27 \%$.

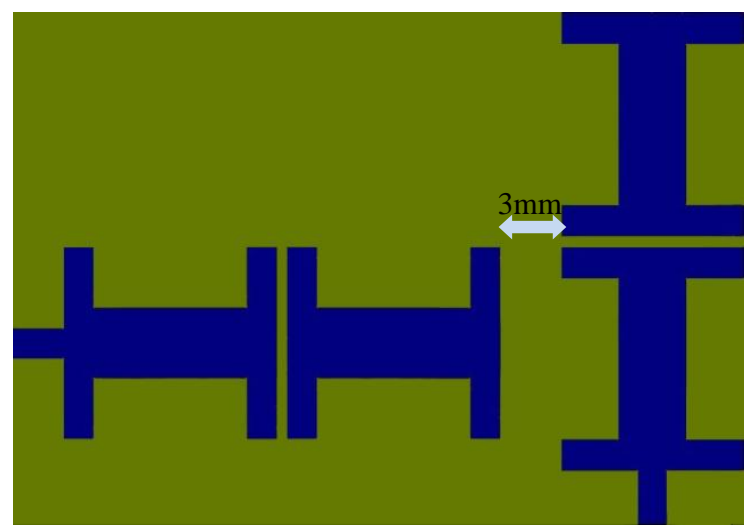

Fig2. Two elements MIMO antennas with parasitic elements

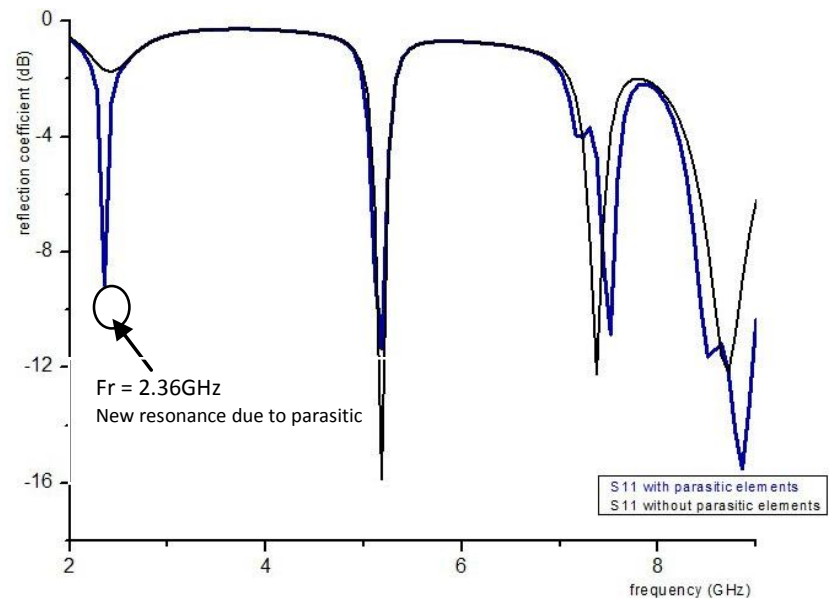

Fig.3 (a) the reflection coefficient with and without parasitic elements

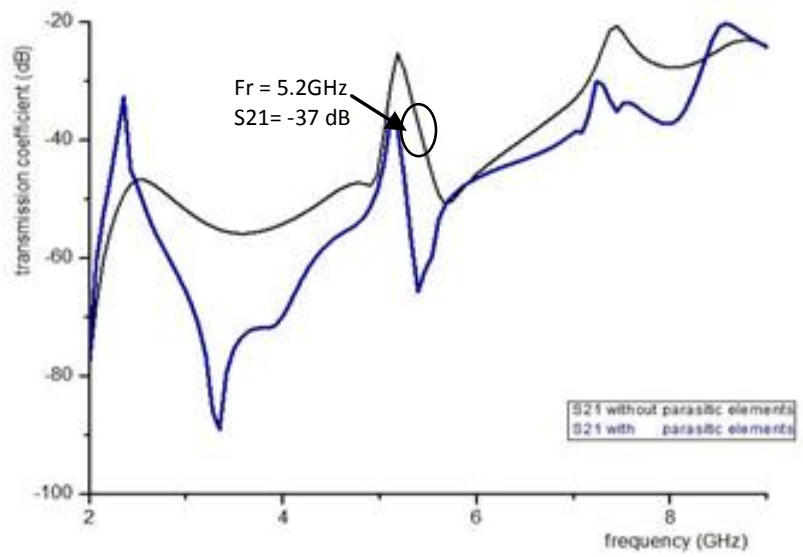

Fig.3 (b) the transmission coefficient with and without parasitic elements

Fig.4 shows the simulated S-parameters of our proposed $(2 * 2)$ MIMO system.

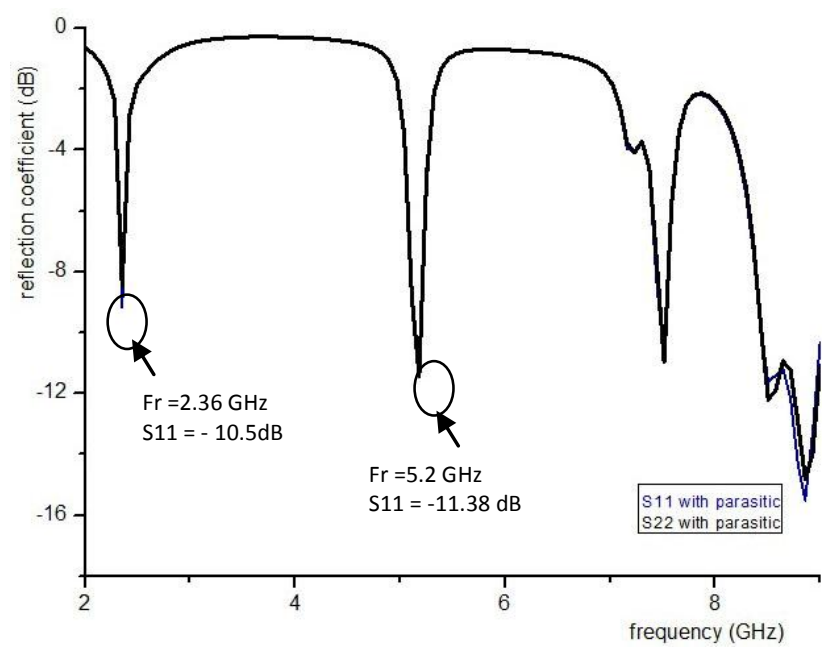

Fig.4. Two elements MIMO with parasitic elements simulation results 
From this figure, the reflection coefficients of the first and second antennas are concurrent at the resonance frequencies $2.36 \mathrm{GHz}, 5.2 \mathrm{GHz}$ and $8.55 \mathrm{GHz}$ which may be used as a reciprocal system (transmitter or receiver). The summary of these results is shown table 1 , which represents the resonance frequencies at $2.36 \mathrm{GHz}, 5.2 \mathrm{GHz}$, and $8.55 \mathrm{GHz}$ with reflection coefficients $-10 \mathrm{~dB},-11.38 \mathrm{~dB},-11.29 \mathrm{~dB}$ and mutual coupling $-34 \mathrm{~dB},-37 \mathrm{~dB}$ and $-21 \mathrm{~dB}$ respectively.

Table 1

\begin{tabular}{|c|c|c|c|c|c|c|}
\hline \multirow{2}{*}{$\begin{array}{c}S \\
(\mathrm{~mm})\end{array}$} & \multicolumn{2}{|c|}{$\operatorname{Fr}(\mathrm{GHz})$} & \multicolumn{2}{c|}{ S11(dB) } & \multicolumn{2}{c|}{ S21(dB) } \\
\cline { 2 - 7 } & Simul. & Meas. & Simul. & Meas. & Simul. & Meas. \\
\hline 3 & 2.36 & 2.39 & -10.58 & -11.43 & -34 & -38.67 \\
\hline 3 & 5.2 & 5.33 & -11.38 & -11.43 & -37 & -38.13 \\
\hline 3 & 8.55 & 8.72 & -11.29 & -16.8 & -21 & -21.56 \\
\hline
\end{tabular}

The radiation patterns of the proposed antenna at the resonant Frequencies are shown in Fig5. The shape of the pattern is the characteristics of such patch antennas.

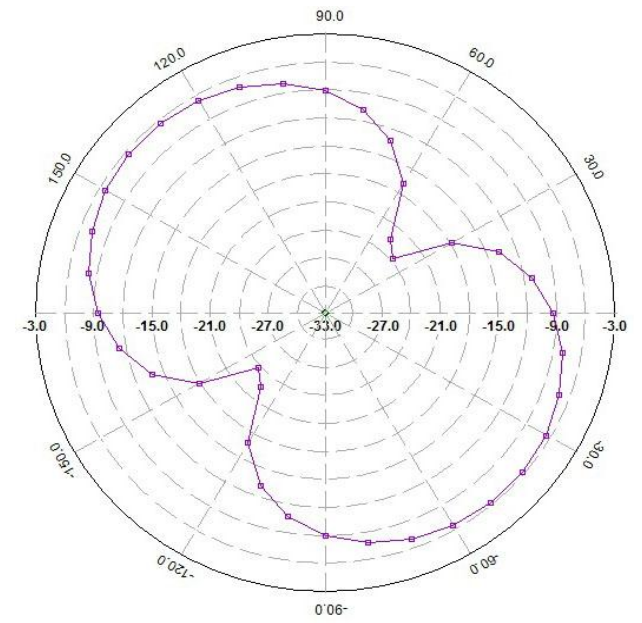

Fig5. (a) Radiation Pattern at 5.2GHz

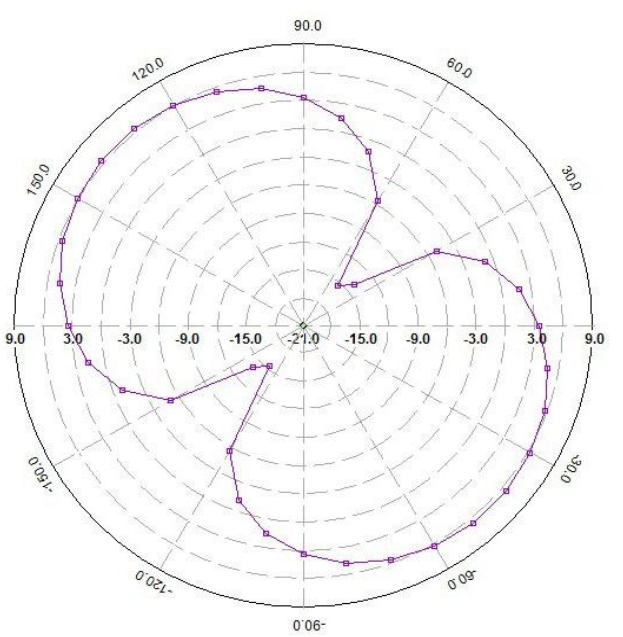

Fig5. (b) Radiation Pattern at 2.36GHz
To verify the simulation results we fabricated specimens of the proposed antenna structure and a photograph of such specimens is shown in Fig.6

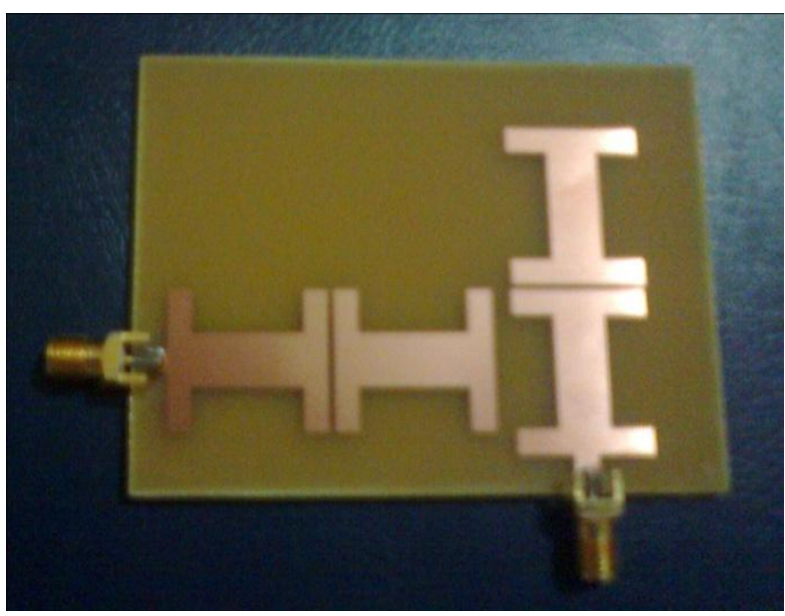

Fig6. The fabricated specimen of the proposed system

Typical measurement results of the specimens are shown in Fig.7.The major measured results of the antenna are also given in table 1 for sake of comparison with the simulated results. It is clear from the table that there is good agreement between the measured and simulated results.

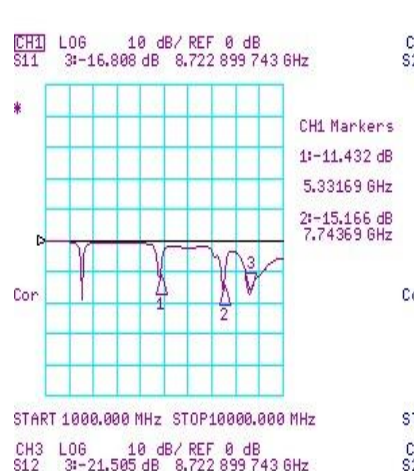

4 0ot 2012 11:38:27

$\begin{array}{lll}\text { CH2 } & \text { LOG } 10 \mathrm{~dB} / \mathrm{REF} \text { O dB } \\ \mathrm{S} 21 & 3:-21.563 \mathrm{~dB} & 8.722899743 \mathrm{GHz}\end{array}$

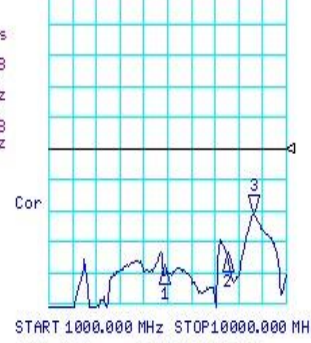

CH2 Markers $1:-38.090 \mathrm{~dB}$ $33169 \mathrm{GHz}$ $2:-33.852 \mathrm{~dB}$
$7.74369 \mathrm{GHz}$

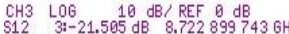
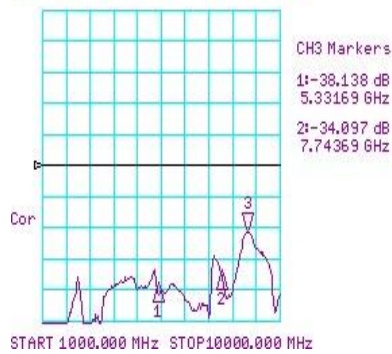

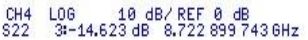

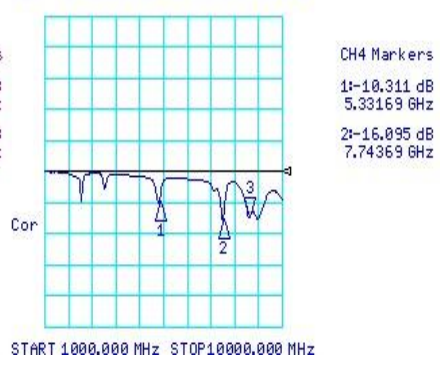

Fig.7 the measurements results of the proposed system

Finally the correlation coefficient of the MIMO antenna is estimated. It is calculated using the formula below and the results are plotted in Fig 8. From the plot, it can be observed that the correlation coefficient is minimized at the resonant frequencies.

$$
\rho=\frac{\left|s_{11}^{*} s_{12}+s_{21}^{*} s_{22}\right|^{2}}{\left(1-\left|s_{11}\right|^{2}-\left|s_{21}\right|^{2}\right)\left(1-\left|s_{22}\right|^{2}-\left|s_{12}\right|^{2}\right)}
$$




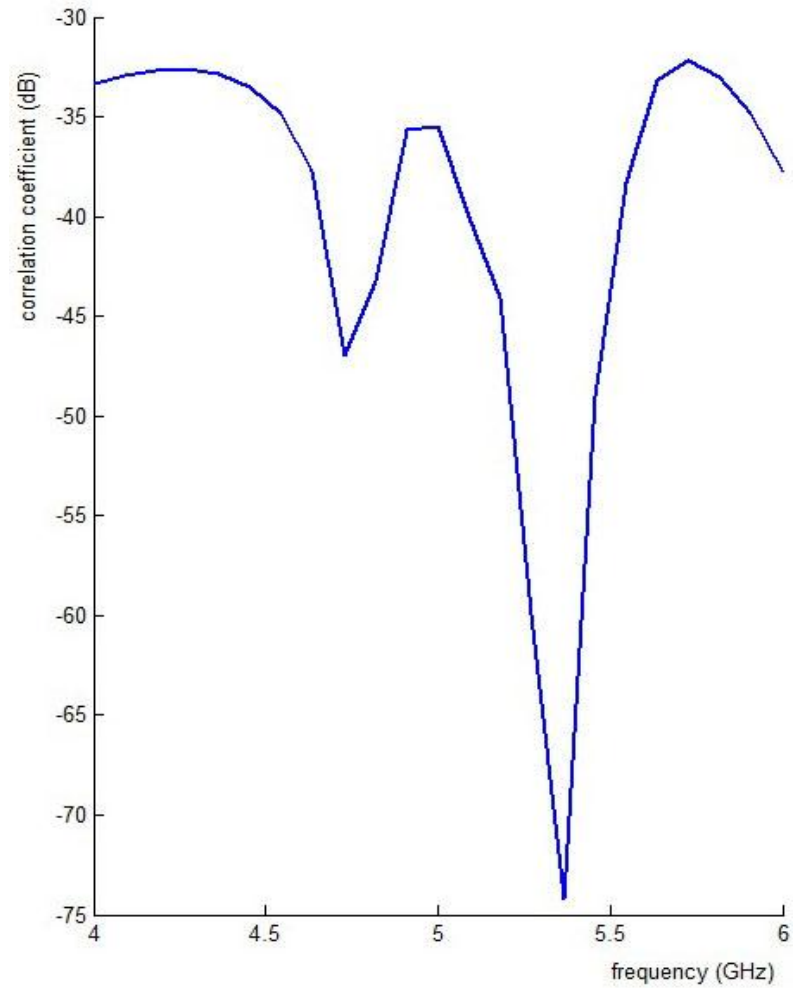

Fig.8. the Correlation Coefficient

\section{CONCLUSION}

In this paper, we firstly introduced two elements MIMO antennas with polarization diversity. The proposed MIMO system resonates at $5.2 \mathrm{GHz}$ and $7.38 \mathrm{GHz}$ frequencies with isolation properties $(\mathrm{S} 21 \leq-26 \mathrm{~dB})$. Secondly a two element MIMO system is proposed using a parasitic elements employing orthogonal polarization diversity. The proposed design resonates at $2.36 \mathrm{GHz}, 5.2 \mathrm{GHz}$ and $8.55 \mathrm{GH}$. We notice that, an addition of the parasitic elements adds new resonance frequencies at $2.36 \mathrm{GHz}$ and $8.55 \mathrm{GHz}$. Also the existence of the parasitic elements improves the decoupling characteristics between the antenna elements, where the mutual coupling becomes less than $-37 \mathrm{~dB}$. These characteristics are well suited for all $4 \mathrm{G}$ MIMO applications.

\section{REFERENCES}

[1] Foschini, G. J. and M. J. Gans, "On limits of wireless communications in a fading environment when using multiple antennas," Wirel. Pers. Commun., Vol. 6, No. 3, 311-335, 1998 .

[2] T.Svantesson."On the Capacity and Correlation of MultiAntenna Systems Employing Multiple Polarizations"Proceedings of IEEE Antennas and Propagation Symposium vol. 3, pp. 202-205, June 2002

[3] Abouda, A. A. and S. G. H" aggman, "Effect of mutual coupling capacity of MIMO wireless channels in high SNR scenario," Progress In Electromagnetics Research, PIER 65, 27-40,

[4] Angus C. K. Mak. Corbett R. Rowell, and Ross D. Murch, "Isolation Enhancement between Two Closely Packed Antennas", IEEE Trans Antennas Propag, vol. 56, No.11, pp.3411-3419, 2008

[5] Shin-Chang Chen, Yu-Shin Wang, and Shyh-Jong Chung, "A Decoupling Technique for Increasing the Port Isolation Between Two Strongly Coupled Antennas" IEEE Trans Antennas Propag,vol.56, No.12, pp.36503658, 2008.

[6] Jussi Rahola, Jani Ollikainen, "Removing the effect of antenna matching in isolation analyses using the concept of electromagnetic isolation", Proc. 2008 International Workshop on Antenna Technology, Chiba, Japan, pp. 554-557, March 4-6,2008.

[7] K. Jagadeesh Babu. "A Multi Slot Patch Antenna for $4 \mathrm{G}$ MIMO Communications," International Journal of Future Generation Communication and Networking Vol. 4, No. 2, June, 2011.

[8] Zhengyi Li, Zhengwei Du, Masaharu takahashi. "Reducing mutual coupling of MIMO Antennas with parasitic elements for mobile terminals," IEEE transactions Antennas and propagation Vol. 60, No. 2, February, 2012. 